\title{
Correction to: Inhibition of Anthracycline Alcohol Metabolite Formation in Human Heart Cytosol: A Potential Role for Several Promising Drugs
}

\author{
In the above article [Mordente A, Silvestrini A, Martorana G E, Tavian D, and Meucci E (2015) Drug \\ Metab Dispos 43:1691-1701; DOI: https://doi.org/10.1124/dmd.115.065110], information regarding the \\ co-corresponding author was omitted. \\ The following co-corresponding information should have been included:
}

Co-corresponding author: Andrea Silvestrini, Institute of Biochemistry and Clinical Biochemistry, School of Medicine, Catholic University, Largo F. Vito 1, 00168, Rome, Italy. E-mail: Andrea.Silvestrini@ unicatt.it. All versions of the article are replaced with the issuance of this erratum.

The compositor apologizes for any inconvenience. 